\title{
Resveratrol inhibits the mTOR mitogenic signaling evoked by oxidized LDL in smooth muscle cells
}

\author{
Paula M. Brito ${ }^{a}$, Raphaël Devillard ${ }^{b}$, Anne Nègre-Salvayre ${ }^{\mathrm{b}, \mathrm{c}, *}$, Leonor M. Almeida $^{\mathrm{a}}$, \\ Teresa C.P. Dinis ${ }^{a}$, Robert Salvayre ${ }^{b, c}$, Nathalie Augé ${ }^{b}$ \\ a Laboratory of Biochemistry, Faculty of Pharmacy, and Center for Neurosciences and Cell Biology, University of Coimbra, Coimbra, Portugal \\ ${ }^{b}$ INSERM-U858, Institut Louis Bugnard CHU Rangueil, Toulouse, France \\ ${ }^{\text {c } F a c u l t e ́ ~ d e ~ M e ́ d e c i n e ~ R a n g u e i l, ~ T o u l o u s e, ~ F r a n c e ~}$
}

\section{A R T I C L E I N F O}

\section{Article history:}

Received 19 June 2008

Received in revised form 24 October 2008

Accepted 4 November 2008

Available online $\mathrm{xxx}$

\section{Keywords:}

Resveratrol

PDK1

Akt

Oxidized LDL

Smooth muscle cells

Proliferation

\begin{abstract}
A B S T R A C T
Objectives: Smooth muscle cell (SMC) proliferation is a major feature in atherosclerosis, since it contributes to the formation of the fibrous cap, thus to plaque stability, but also to arterial stenosis and post-angioplasty restenosis. Among the various mitogenic signaling pathways involved in SMC proliferation, the mTOR pathway regulates both the cell cycle and cell growth. Resveratrol, a polyphenolic compound from grapes and red wine, has potential anti-atherogenic and anti-cancer properties. This work was designed to investigate the activation of the MTOR pathway by the proatherogenic oxidized LDL (oxLDL) in SMC, and the potential inhibitory effect of resveratrol.

Results: mTOR and its downstream target p70S6 kinase are phosphorylated and activated by mitogenic concentrations of oxLDL $(50 \mu \mathrm{g} / \mathrm{ml})$, and are involved in SMC proliferation, as assessed by the inhibitory effect of the mTOR inhibitor rapamycin. The activation of mTOR signaling by oxLDL, requires the upstream activation of PI3K and Akt, as assessed by the inhibitory effect of the PI3K inhibitor Ly294002 on mTOR activation and DNA synthesis. Resveratrol blocked the oxLDL-induced phosphorylation and activation of the PI3K/Akt/mTOR/p70S6K pathway and strongly inhibited both the DNA synthesis and proliferation of SMC. This activity is independent of the anti-oxidant effect and of AMPK activation by resveratrol.

Conclusion: These data indicate that the mTOR pathway is activated by oxLDL via PI3K/PDK1/Akt, and is required for SMC proliferation. Resveratrol blocks specifically this pathway, thereby inhibiting oxLDLinduced SMC proliferation. These data highlight a new property for resveratrol that could contribute to the general anti-atherogenic properties of this polyphenol.
\end{abstract}

(c) 2008 Elsevier Ireland Ltd. All rights reserved.

\section{Introduction}

Vascular smooth muscle cells proliferation is a key process in the pathogenesis of atherosclerosis as it underlies the formation of fibro-atheroma plaques, a hallmark of the disease [1]. Oxidized low density lipoproteins (oxLDL) are involved in the formation of fatty streaks and exhibit various biological properties, including vascular smooth muscle cells proliferation, that are potentially implicated in the evolution towards more advanced lesions [2]. The mitogenic effect of oxLDL depends on the activation of various signaling

Abbreviations: LDL, low density lipoproteins; SMC, smooth muscle cells; oxLDL, oxidized low density lipoprotein; FCS, fetal calf serum; PI3K, phosphoinositide 3 kinase; mTOR, mammalian target of rapamycin.

* Corresponding author at: INSERM U858-Team 10 - IFR-31, Institut Louis Bugnard, CHU Rangueil, 1 avenue Jean Poulhès, BP84225 - 31432 Toulouse Cedex 4, France. Tel.: +33 5613228 08; fax: +33561322084.

E-mail addresses: anne.negre-salvayre@inserm.fr (A. Nègre-Salvayre), nathalie.auge@inserm.fr (N. Augé). pathways including the sphingolipid pathway leading to ERK1/2 phosphorylation, and the EGF receptor/PI3K/Akt pathway involved in survival [3-5]. Beside its role in cell survival, the PI3K/Akt pathway is a key trigger of mTOR signaling. The Ser/Thr kinase mTOR is implicated in spatial and temporal aspects of cell growth, controlling translation and cell cycle progression by phosphorylating 4EBP1 protein family and S6 protein kinases [6,7]. The activation of mTOR through PI3K/Akt involves a direct Akt-mediated phosphorylation of mTOR at Ser2448 and Thr2446 [8,9]. Although mTOR has been implicated in cardiovascular diseases and more particularly in cardiac hypertrophy, its role in oxLDL-induced SMC proliferation is still unknown.

Resveratrol (3,4,5-trihydroxystilbene) is a natural phytoalexin found in grapes and red wine. A growing body of evidences in the literature reports a protective effect for resveratrol as antioxidant, anti-inflammatory, anti-thrombotic and anti-proliferative agent, thereby supporting a role for this agent in the cardioprotective effects observed by a moderate consumption of red wine [10-12]. Several molecular mechanisms and signaling pathways

0021-9150/\$ - see front matter @ 2008 Elsevier Ireland Ltd. All rights reserved. doi:10.1016/j.atherosclerosis.2008.11.011 
have been reported to underly resveratrol ability to prevent SMC proliferation. Indeed, resveratrol has been reported to inhibit the cellular signaling exerted by various agents such as growth factors, TNF- $\alpha$, angiotensin II or oxLDL [13-17]. Liu and Liu [18] have shown that resveratrol hindered SMC proliferation mediated by oxLDL through reduction of ROS production and ERK1/2 activation. Moreover, resveratrol may also interrupt the Akt pathway induced by angiotensin II, EGF and PDGF, but not that elicited by serum [16,17].

The aim of this work was to investigate whether the mTOR pathway is required for the mitogenic effect of oxLDL in SMC and to evaluate whether resveratrol could modulate this potentially pathogenic signaling.

\section{Materials and methods}

\subsection{Reagents}

$\left[{ }^{3} \mathrm{H}\right]$ thymidine $(5 \mathrm{Ci} / \mathrm{mmol})$ was obtained from Amersham (Les Ulis, France), resveratrol from Extrasynthese (Genay, France), Ly294002, PD98059 and rapamycin from Calbiochem (Darmstadt, Germany), FPA-124 (Akt inhibitor) from Echelon/Tebu (Le Perray, France) and the other reagents were from VWR (France) or Sigma (France). Inhibitors were stored in DMSO at $-20^{\circ} \mathrm{C}$. For the experiments, the final concentration in DMSO did not exceeded $0.1 \%$. A same amount of DMSO (vehicle) was used in the controls without inhibitors. Rabbit polyclonal antibodies against phospho-mTOR (Ser2448), phospho-PDK1 (Ser241), phospho-MAPK, phospho-p70S6K (Thr389), phospho-Akt and $\beta$ actin were from Cell Signaling (Beverley, MA, USA); AMPK, phosphoAMPK and ERK2 were from Santa Cruz Biotechnology (Santa Cruz, CA, USA). SiRNA specific for AMPK (smartpool no. L-005361), were from Dharmacon (ThermoFisher Scientific, Perbio/Eurotech).

\section{2. $L D L$ preparation and oxidation}

Human low-density lipoproteins (LDL) were isolated from pooled fresh plasma and stored at $4{ }^{\circ} \mathrm{C}$ under nitrogen until use, as previously described [3]. LDL were oxidized by UV-C irradiation, and the extent of oxidation was evaluated by monitoring the formation of thiobarbituric acid reactive substances (TBARS), as previously reported [3]. Under the standard conditions used here, oxLDL contained 2.5-6 nmol TBARS/mg protein. LDL protein was quantified according to Lowry procedure.

\subsection{Cell culture and treatment}

Rabbit femoral smooth muscle cells (SMC) were from ATCC (Camden, NJ, USA) and were cultured in RPMI-1640 medium supplemented with $10 \%$ fetal calf serum (FCS), $100 \mathrm{U} / \mathrm{ml}$ penicillin, $100 \mu \mathrm{g} / \mathrm{ml}$ streptomycin at $37^{\circ} \mathrm{C}$, in a humidified atmosphere of $5 \%$ $\mathrm{CO}_{2}$. Cells were starved in RPMI medium containing 0.5\% FCS for $24 \mathrm{~h}$ before the experiments.

\subsection{Transfection of siRNA}

AMPK (smartpool no. L-005361) and scrambled siRNA were purchased from Dharmacon (Lafayette, CO). SMC were transfected with $100 \mathrm{nM}$ double strand siRNA in Optimem medium (Invitrogen) mixed with Hiperfect, under supplier's instructions as described [19]. $48 \mathrm{~h}$ after transfection, cells were incubated in RPMI containing $10 \%$ FCS for $24 \mathrm{~h}$. Then SMC were incubated for $12 \mathrm{~h}$ in RPMI containing $0.5 \% \mathrm{FCS}$, before experiments.

\subsection{Cell proliferation and DNA synthesis}

DNA synthesis was evaluated by $\left[{ }^{3} \mathrm{H}\right]$ thymidine incorporation into DNA, under the previously used conditions [5].

Cell counting was performed as previously described, using a Z1 Beckman cell counter [20].

\subsection{Cell viability, apoptosis and necrosis}

Cytotoxicity was evaluated using a two-color fluorescence live/dead viability/cytotoxicity assay, using two vital fluorescent dyes, $0.6 \mu \mathrm{M}$ SYTO-13 (a permeant DNA intercalating green-colored probe) and $15 \mu \mathrm{M}$ propidium iodide (a non-permeant intercalating red probe), as described [5]. Necrotic and apoptotic cells were counted by using an inverted fluorescence microscope (Fluovert FU, Leitz).

\subsection{Western blot experiments}

Time-course experiments of ERK1/2, Akt, mTOR and p70S6K1 phosphorylation were analyzed by Western blot after incubation with oxLDL and inhibitors, PD098059, Ly294002 or rapamycin and resveratrol. After stimulation, cells were washed and scraped in PBS, then disrupted at $4{ }^{\circ} \mathrm{C}$ in $20 \mathrm{mM}$ HEPES, $1 \mathrm{mM}$ EDTA, $1 \mathrm{mM}$ $\mathrm{Na}_{3} \mathrm{VO}_{4}, 250 \mathrm{mM}$ sucrose, $5 \mu \mathrm{M}$ digitonine, $1 \mathrm{mM}$ DTT, $1 \mathrm{mM}$ PMSF, for $15-30 \mathrm{~min}$. After two cycles of clarification at $2500 \times \mathrm{g}$ for $5 \mathrm{~min}$, supernatant was centrifuged at $12,000 \times \mathrm{g}$ for $15 \mathrm{~min}$ (Beckman Optima) and used for Western blot experiments in the previously reported conditions [5].

\subsection{Intracellular reactive oxygen species increase determination}

Time-course generation of intracellular ROS was measured after loading SMC with the permeant H2DCFDA probe, as previously reported [21].

\subsection{PI3 kinase activity evaluation}

PI3K activity was determined on phosphotyrosine protein immunoprecipitates according to Burgering et al. [22] using $20 \mu \mathrm{g}$ phosphatidylinositol in a 30-mM HEPES buffer containing $200 \mu \mathrm{M}$ adenosine and $1 \mu \mathrm{Ci}\left[\gamma-{ }^{33} \mathrm{P}\right] \mathrm{ATP}, 40 \mu \mathrm{M}$ ATP and $30 \mathrm{mM} \mathrm{MgCl}_{2}$ per assay. $\left[{ }^{33} \mathrm{P}\right]$ phosphoinositides were localized by autoradiography, scraped off, and counted by liquid scintillation.

\subsection{Statistical analysis}

All data were expressed as mean \pm S.E.M. of at least three independent assays, each one in duplicate. Differences between groups were analysed by one-way analysis of variance (ANOVA) followed by Tukey's or Dunnet's post-test. $p<0.05$ was considered statistically significant.

\section{Results}

\subsection{Mitogenic concentration of oxLDL trigger the activation of the PI3K/PDK1/Akt/mTOR/p70S6 kinase pathway}

OxLDL induce a dose-dependent SMC proliferation, with a maximal mitogenic effect between 50 and $100 \mu \mathrm{g}$ apoB $/ \mathrm{ml}$, as assessed by increased DNA synthesis (determined as $\left[{ }^{3} \mathrm{H}\right]$ thymidine incorporation in the DNA), and increased cell number (Fig. 1A). It is to note that mitogenic oxLDL concentration $(50 \mu \mathrm{g}$ apoB $/ \mathrm{ml})$ used here was not toxic (apoptotic), in contrast to higher concentrations $(200 \mu \mathrm{g}$ apoB/ml) (Fig. 1B and C), and in agreement with [3]. 

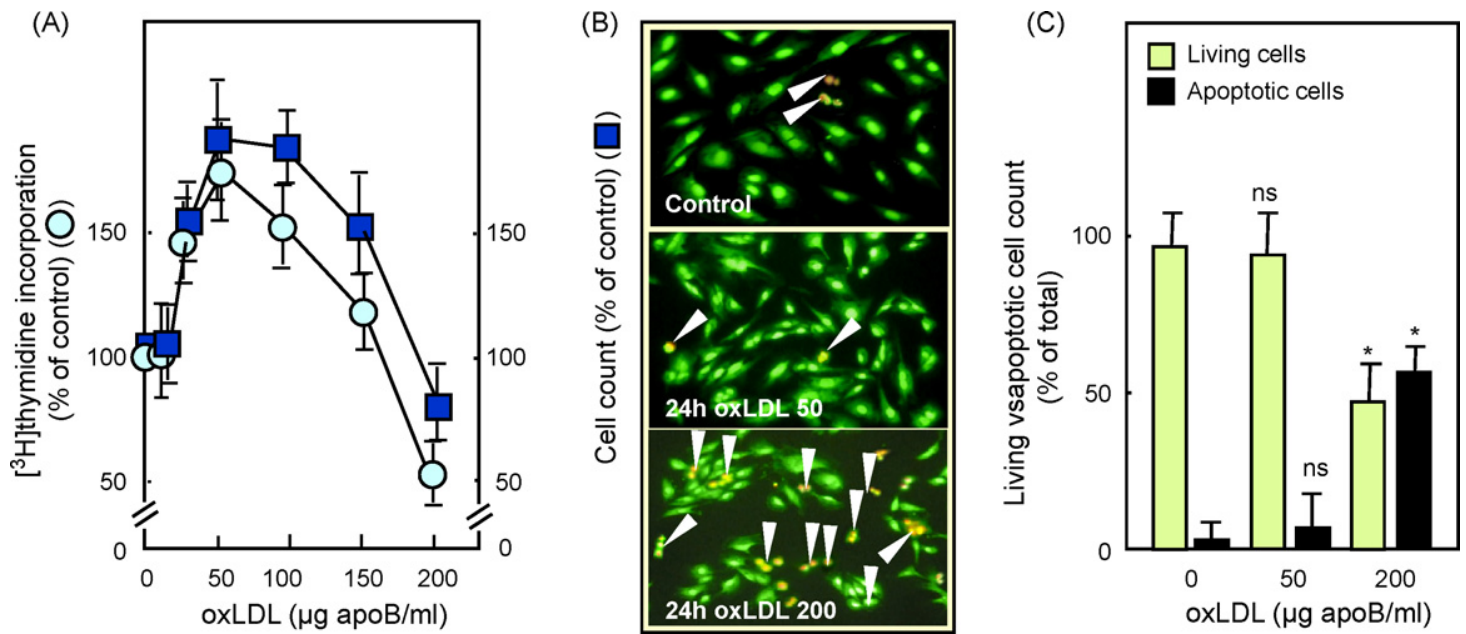

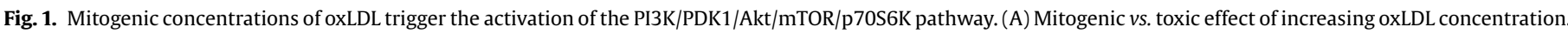

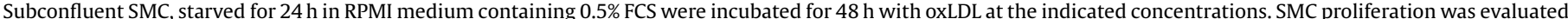

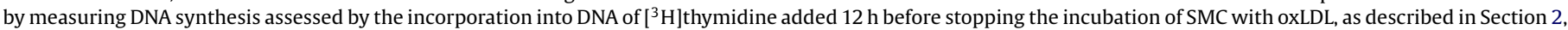

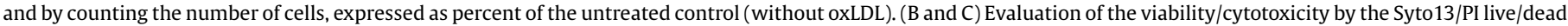

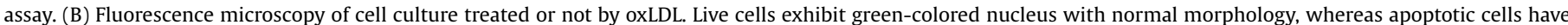

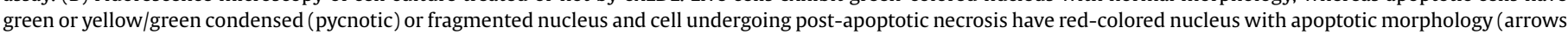

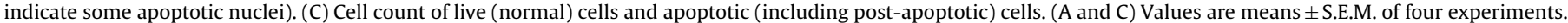

Mitogenic oxLDL concentrations $(50 \mu \mathrm{g}$ apoB $/ \mathrm{ml})$ rapidly induced PI3 kinase activation that started after 30 min incubation with oxLDL and was maintained over $3 \mathrm{~h}$ of stimulation (Fig. 2A). Concomitantly, oxLDL triggered the phosphorylation of PDK1 and Akt (Fig. 2B and C). Since the PI3K/Akt pathway is a known trigger of mTOR [6-9], we investigated whether
mTOR and its downstream target p70S6 kinase (p70S6K) were activated by oxLDL. As shown in Fig. 2D-F, oxLDL induced a time- and dose-dependent phosphorylation of mTOR and p70S6K, which was concomitant to the activation of PI3K/PDK1/Akt, and was optimal for mitogenic oxLDL concentrations $(50 \mu \mathrm{g}$ apoB/ml).
(A)

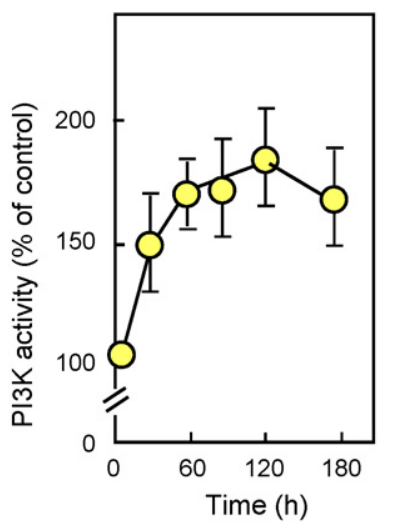

(D)

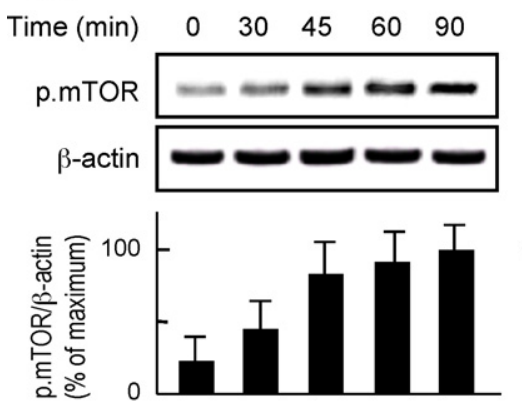

(B)
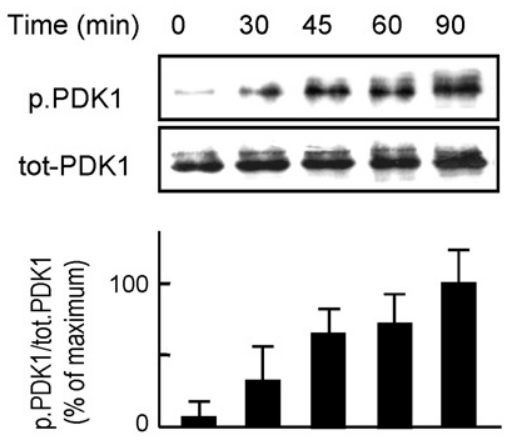

(E) $\quad$ oxLDL $(\mu \mathrm{g} \mathrm{apoB} / \mathrm{ml})$
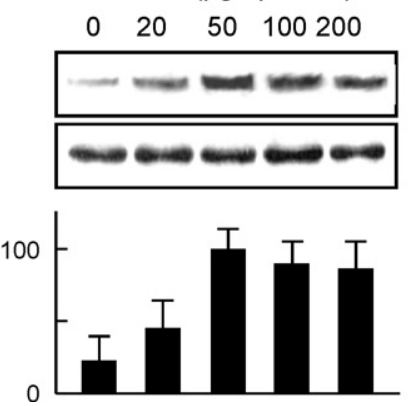

(C)
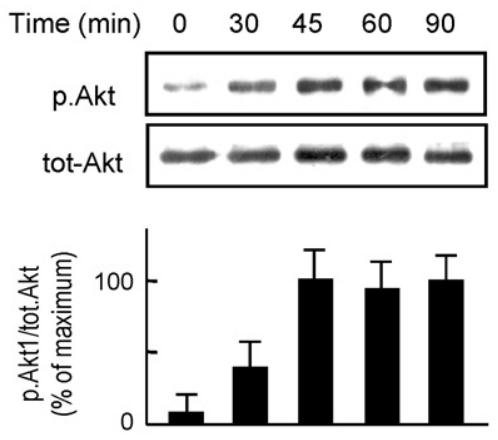

(F)

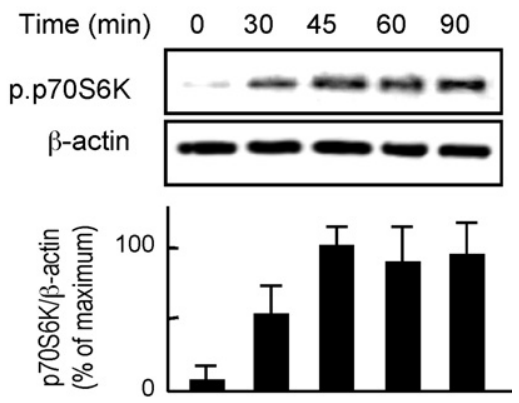

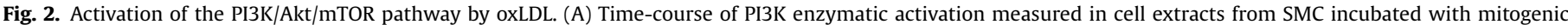

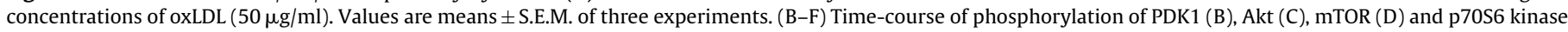

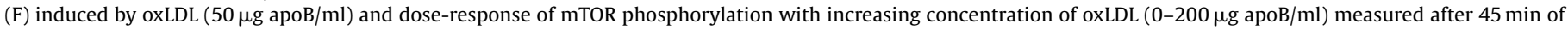
incubation of cells with oxLDL. Each blot is representative of three experiments. Values of densitometric analysis are means \pm S.E.M. of three experiments. 


\subsection{The PI3K/Akt/mTOR pathway is required for the mitogenic effect of oxLDL}

Then, we investigated the potential role of the PI3K/PDK1/Akt system and mTOR activation in SMC proliferation elicited by oxLDL. As shown in Fig. 3A, the pharmacological PI3K inhibitors, Ly294002 and wortmannin, blocked the phosphorylation of Akt. Similarly, the phosphorylation of mTOR and p70S6K was inhibited by Ly294002, thus suggesting that mTOR and p70S6K activation is dependent on PI3K (Fig. 3B and C). The inhibition by rapamycin of p70S6K phosphorylation indicates that p70S6K activation depends on mTOR (Fig. 3C). Rapamycin dose-dependently inhibited DNA synthesis ( $\left[{ }^{3} \mathrm{H}\right]$ thymidine incorporation) (pale bars), and cell proliferation (dark bars), a full inhibitory effect being observed at $10-100 \mathrm{nM}$ (Fig. 3D and E). In cells treated with oxLDL $(50 \mu \mathrm{g}$ apoB $/ \mathrm{ml})$, rapamycin was not toxic up to $100 \mathrm{nM}$ (Fig. 3E and F). SMC proliferation was also completely inhibited by the PI3K inhibitors Ly294002 and wortmannin and by the Akt inhibitor FPA-124 (Fig. 2D and E), without toxic effect (Fig. 3E).

Altogether, these data suggest that SMC proliferation elicited by mitogenic concentration of oxLDL requires the activation of the PI3K/Akt/mTOR pathway.

\subsection{Resveratrol inhibits oxidized LDL-induced SMC proliferation}

Since resveratrol has been shown to inhibit SMC proliferation induced by various agonists [23,24] and regulate cell survival/death [25], we investigated whether resveratrol inhibited the proliferation of SMC by oxLDL and whether it increases or decreases the cytotoxicity of oxLDL.
(A)

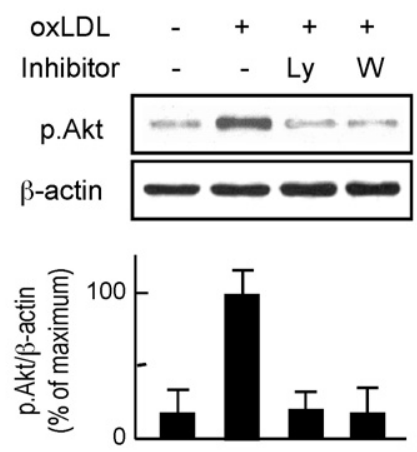

(B)

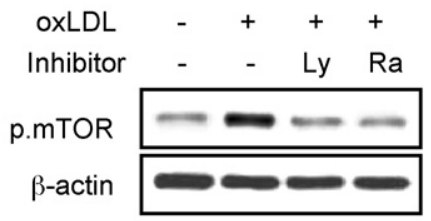

(C)
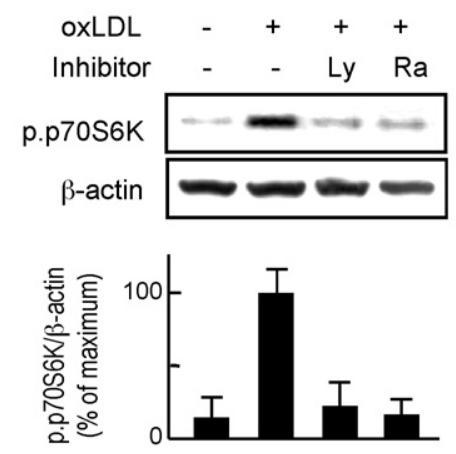
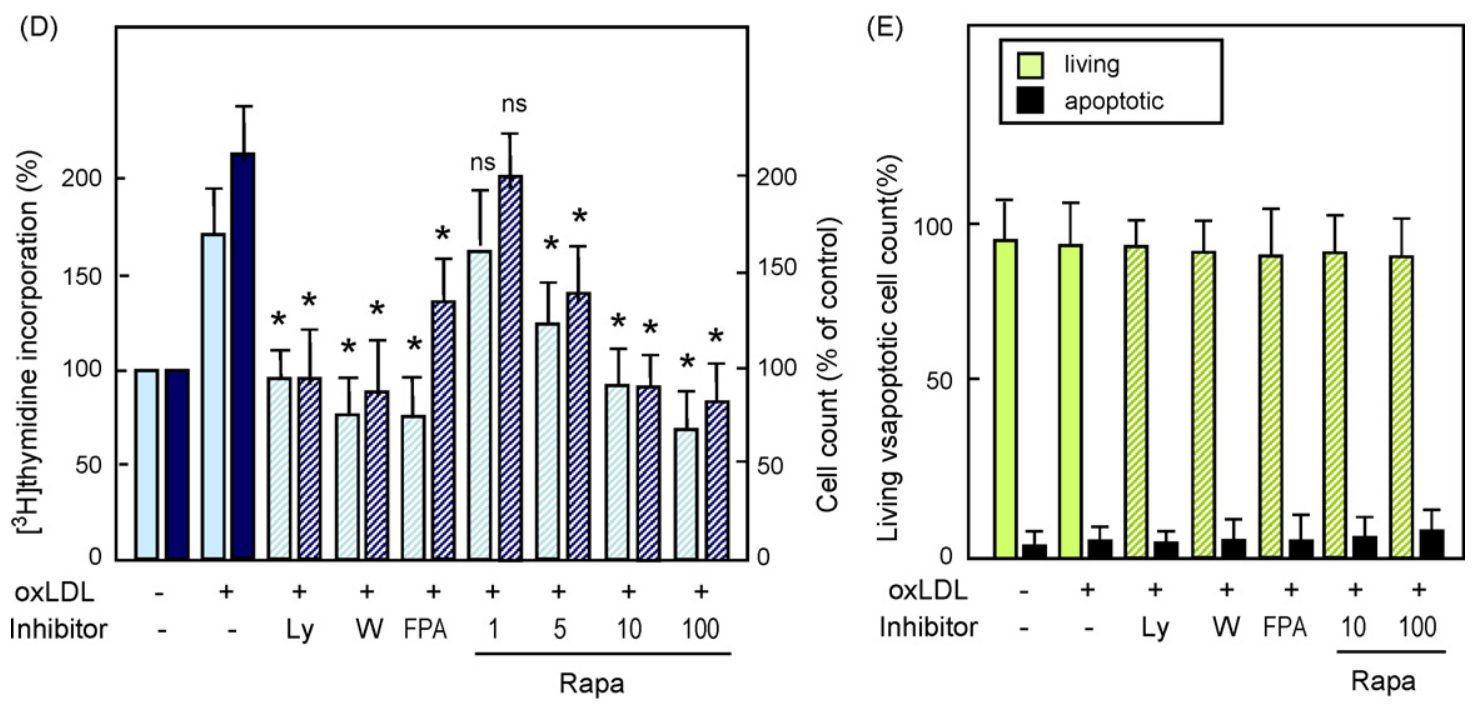

(F)

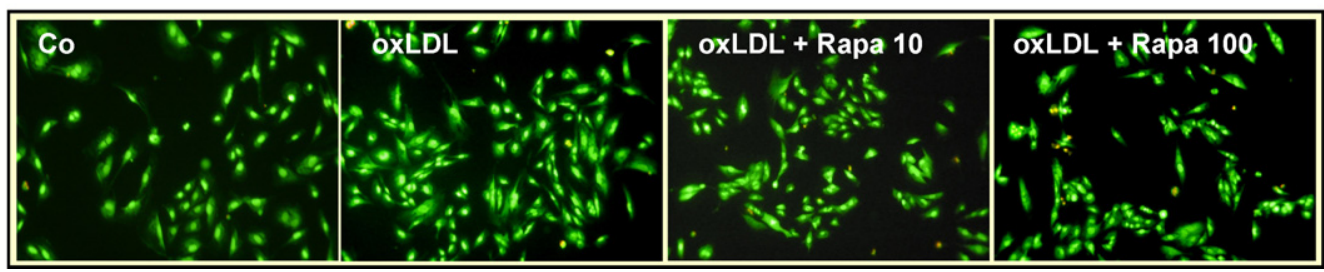

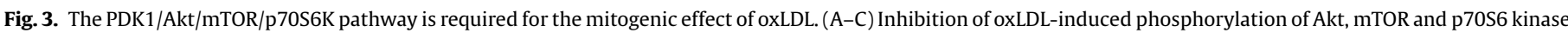

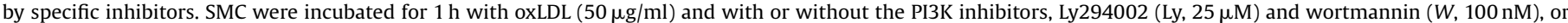

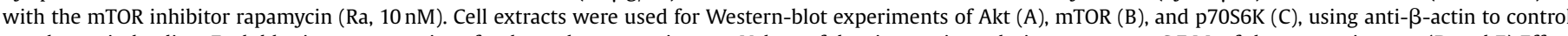

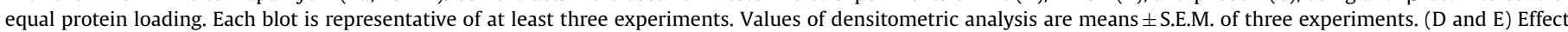

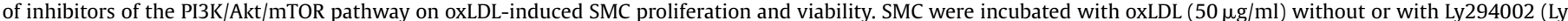

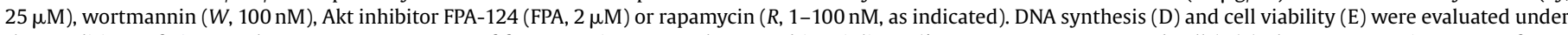

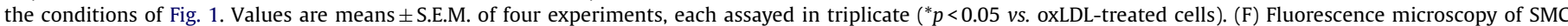
incubated with oxLDL \pm rapamycin and stained with Syto13/P, under conditions of Fig. 1B. 
Dose-response experiments showed that DNA synthesis and cell proliferation were significantly inhibited by $25 \mu \mathrm{M}$ resveratrol (Fig. 4A and B), without any significant apoptotic effect at this concentration (Fig. $4 \mathrm{C}$ and D). It may be noted that, $50 \mu \mathrm{M}$ resveratrol exhibited a slight toxic effect in the presence of oxLDL (Fig. 4C). Interestingly, $25 \mu \mathrm{M}$ resveratrol inhibited almost completely the phosphorylation of ERK1/2, in agreement with its anti-proliferative effect (Fig. 4E), thus suggesting that it inhibits the mitogenic signaling triggered by oxLDL. As resveratrol has been shown to inhibit PI3K/Akt activation by EGF and angiotensin II [17], we investigated whether it was able to inhibit the oxLDLinduced activation of PI3K/Akt/mTor pathway. As shown in Fig. 5, resveratrol inhibited the phosphorylation of PDK1, Akt, mTOR and p70S6K1 induced by oxLDL. This strongly suggests that resveratrol acts on an upstream target in the PI3K/Akt/mTOR signaling pathway.

\subsection{Resveratrol prevents oxLDL-induced SMC proliferation independently of ROS scavenging}

As resveratrol (like other polyphenolic compounds) exhibits anti-oxidant properties, we investigated whether the anti-oxidant effect was involved in the inhibition of the PI3K/Akt/mTOR signaling pathway. OxLDL induced an early rise of intracellular reactive oxygen species (ROS) peaking $1 \mathrm{~h}$ after adding oxLDL to the culture medium (Fig. 6A). The ROS peak was inhibited by $25 \mu \mathrm{M}$ resveratrol, $20 \mathrm{mM}$-acetylcysteine (NAC) or $10 \mu \mathrm{M}$ Trolox (Fig. 6B). In contrast, Akt phosphorylation was only inhibited by resveratrol $(25 \mu \mathrm{M})$, whereas NAC and trolox were not effective (Fig. 6C). This strongly suggests that resveratrol prevents oxLDL-induced Akt phosphorylation probably independent of its anti-oxidant effect, since anti-oxidants such as $\mathrm{N}$-acetylcysteine and trolox did not inhibit Akt phosphorylation by oxLDL (in contrast to resveratrol), while all these agents exhibited similar inhibitory effect on intracellular ROS generation.

\subsection{The inhibitory effect of resveratrol against oxLDL-induced SMC proliferation is independent of AMPK}

Resveratrol has also been shown to activate AMP-activated kinase (AMPK), which could take part in the protective and antiatherogenic properties of polyphenolic compounds [26]. Moreover, the activation of AMPK induces TSC2 and raptor phosphorylation, resulting finally in mTOR inhibition and suppression
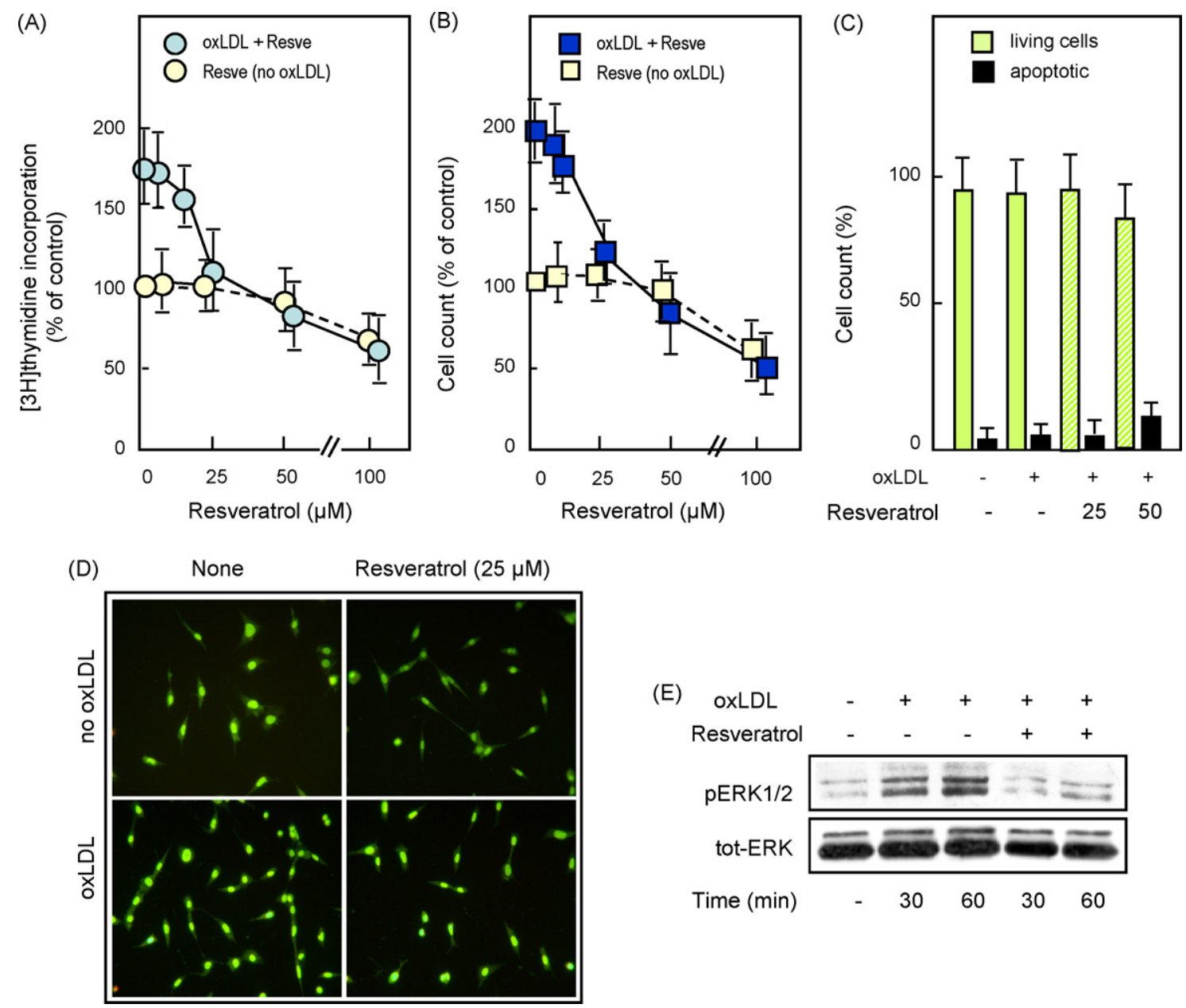

(E)

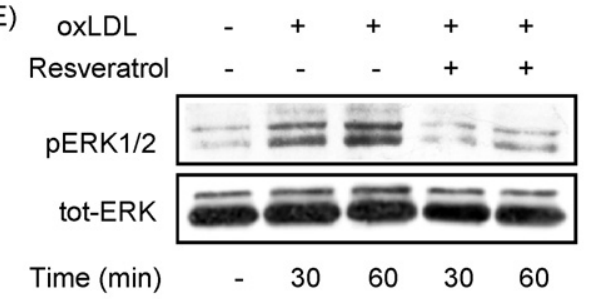

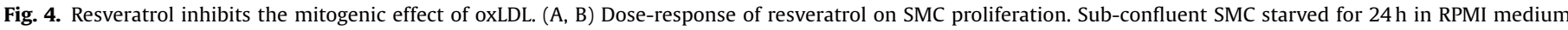

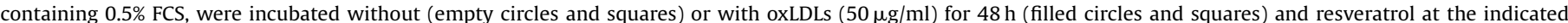

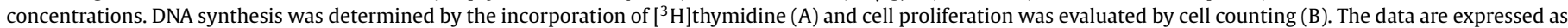

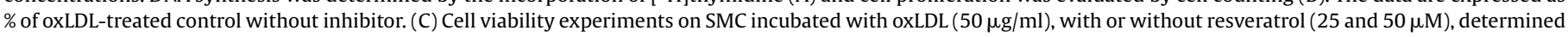

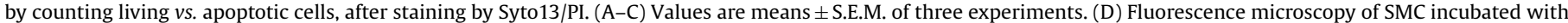

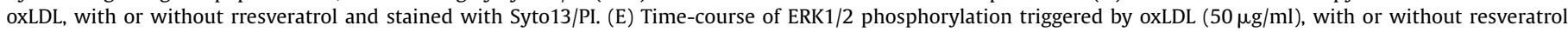

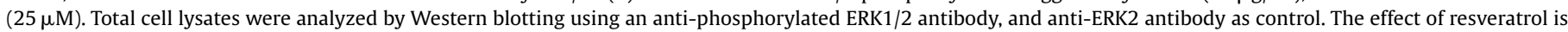
representative of five separate experiments. 
(A)
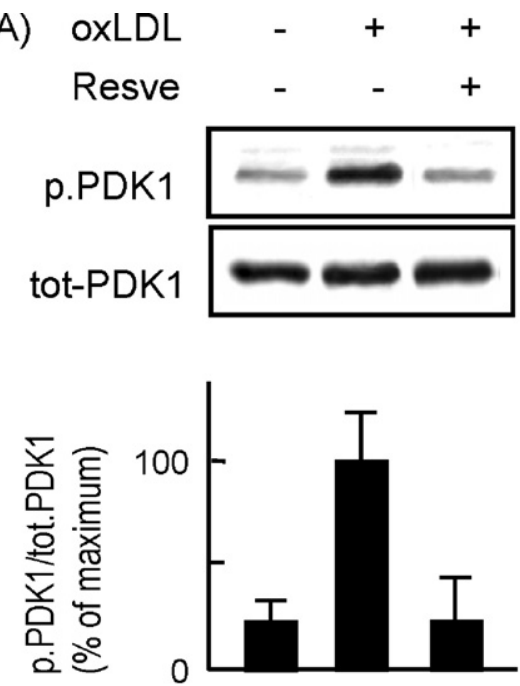

(C)
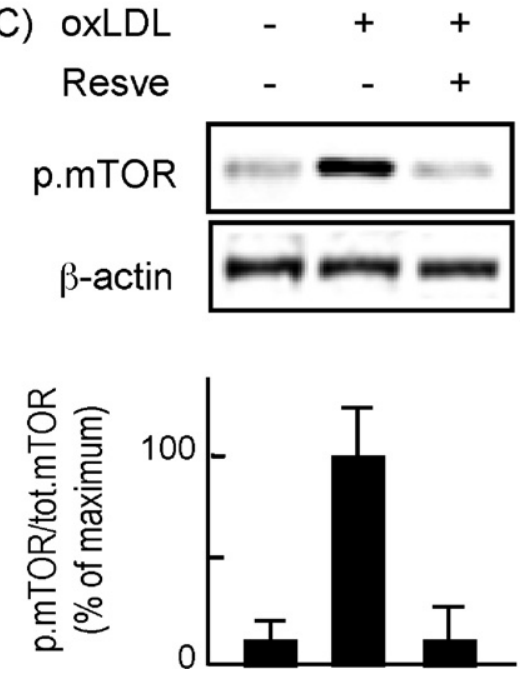

(B)
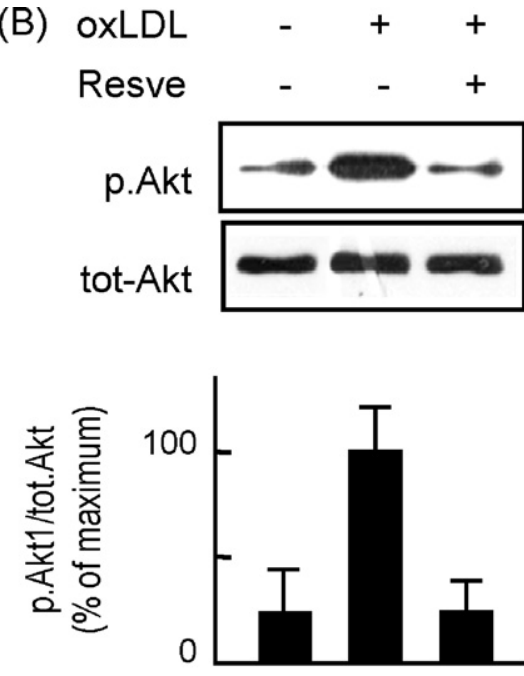
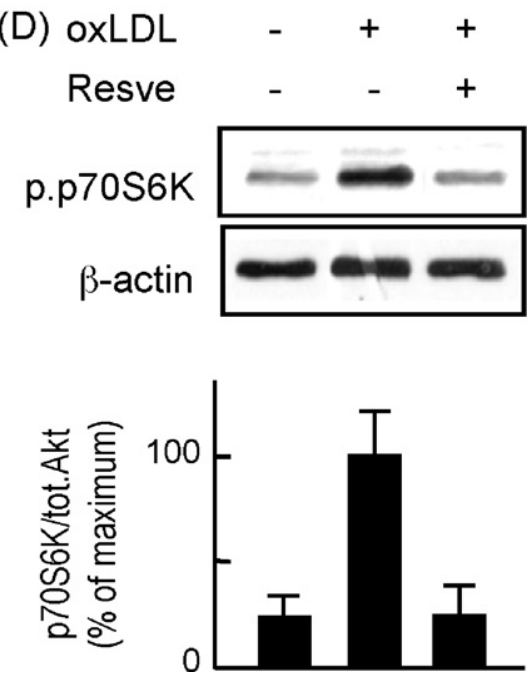

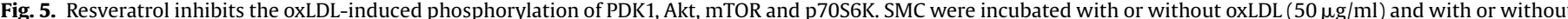

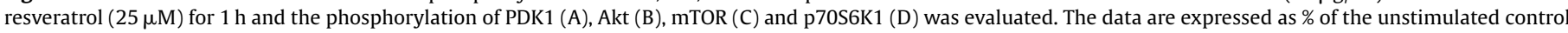
cells. Values of densitometric analysis are means \pm S.E.M. of three experiments.

(A)

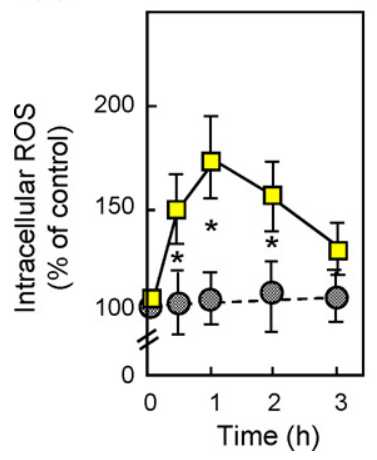

(B)

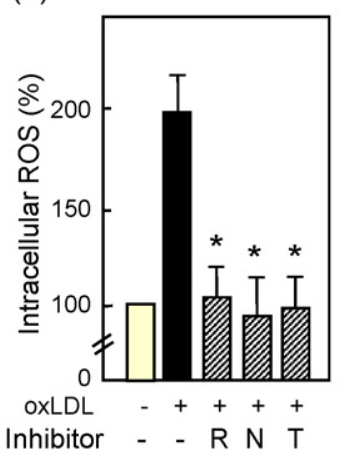

(C)

oxLDL -++++

Inhibitor - $-\mathrm{R} \mathrm{N} \mathrm{T}$

p.Akt $\cdots-\infty$

$\beta$-actin $\longrightarrow$

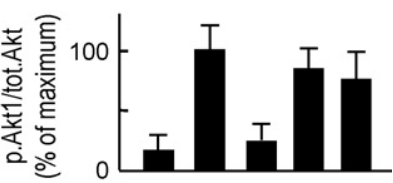

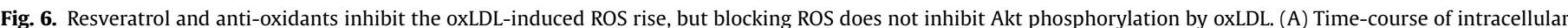

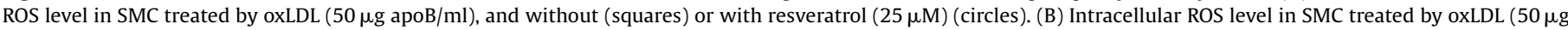

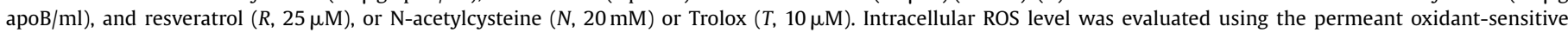

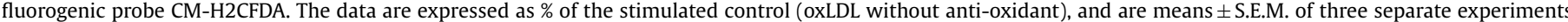

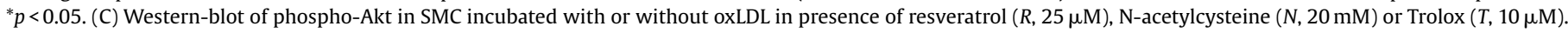
These data are representative of three separate experiments.

Please cite this article in press as: Brito PM, et al. Resveratrol inhibits the mTOR mitogenic signaling evoked by oxidized LDL in smooth muscle cells. Atherosclerosis (2008), doi:10.1016/j.atherosclerosis.2008.11.011 

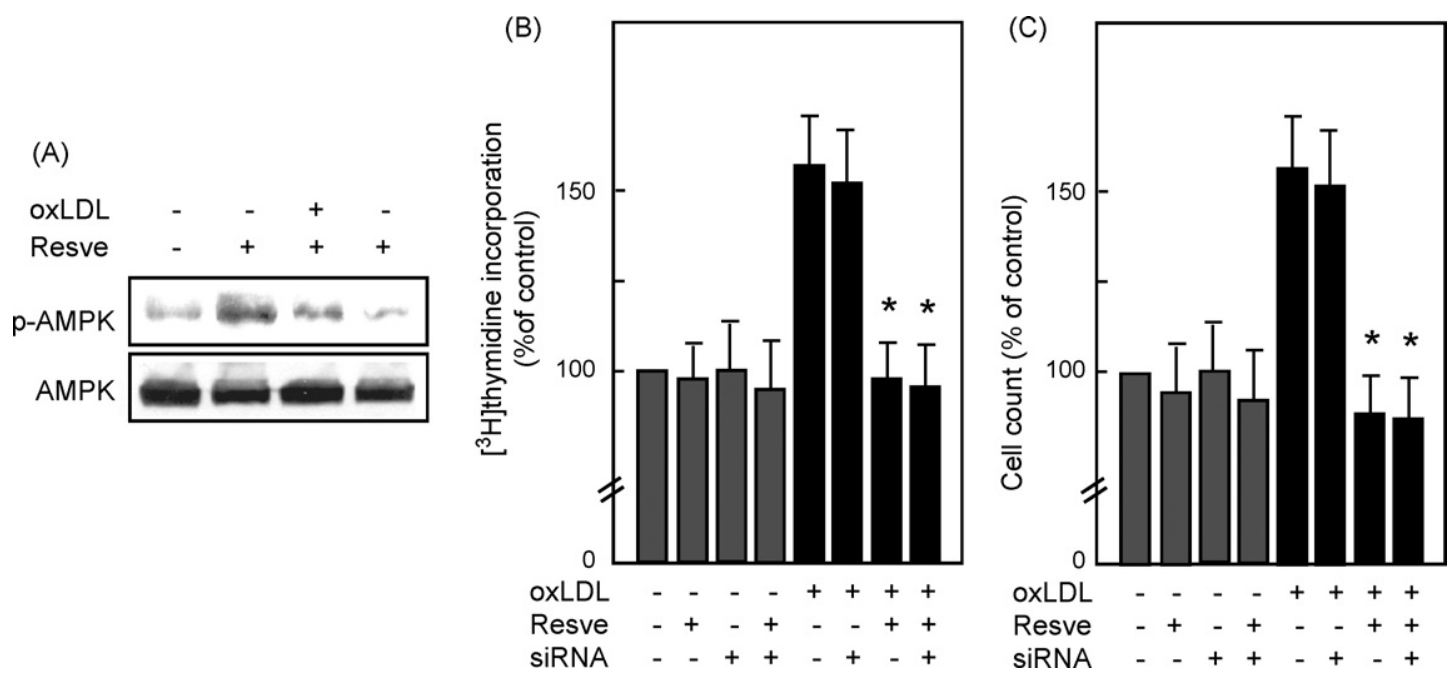

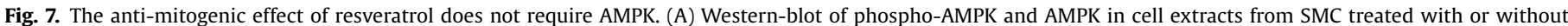

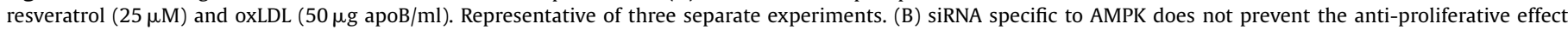

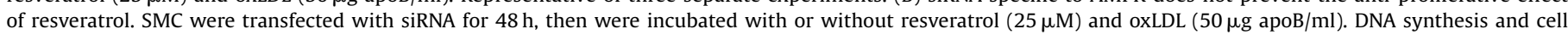
proliferation were evaluated as described in Fig. 1A. Data are means \pm S.E.M. of three separate experiments. ${ }^{*} p<0.05$.

of cell proliferation [27]. This led us to investigate whether AMPK is involved in the anti-proliferative effect of resveratrol.

As expected, resveratrol triggered the activation of AMPK, whereas oxLDL did not trigger AMPK phosphorylation (Fig. 7A). Interestingly, in cells treated with oxLDL and resveratrol, we observed no AMPK phosphorylation, thus suggesting that oxLDL inhibited the resveratrol-induced AMPK activation (Fig. 7A). This strongly suggests that, under our experimental conditions, AMPK is not involved in the inhibitory effect of resveratrol on the PI3K/Akt/mTOR pathway and oxLDL-induced proliferation. It may be noted that compound $C$, an AMPK inhibitor, inhibited oxLDLinduced SMC proliferation, independent of any effect of AMPK. Finally, depletion of AMPK by specific siRNA, induced no significant change on oxLDL-induced SMC proliferation and did not alter the inhibitory effect of resveratrol (Fig. 7B).

Altogether, these data strongly suggest that the inhibition of oxLDL-induced SMC proliferation by resveratrol does not require AMPK.

\section{Discussion}

The data reported here indicate that the mTOR pathway is activated by oxLDL, and is involved in SMC proliferation. Resveratrol, a polyphenolic phytoalexin present in red wine, potently inhibits mTOR signaling and the subsequent SMC proliferation exerted by oxLDL, by interfering specifically with the PI3K/Akt signaling cascade.

Among the various mitogenic and survival pathways activated by oxLDL, we have previously reported that the PI3K/Akt pathway is required for SMC survival and proliferation [5]. This pathway is a trigger of the serine/threonine kinase mTOR, which regulates many cellular processes including cell growth, proliferation, survival, autophagy, and cell metabolism [9]. This is, to our knowledge, the first report showing that mTOR is activated by oxLDL. Moreover, mTOR activation is required for oxLDL-induced DNA synthesis and SMC proliferation, as demonstrated by the inhibitory effect of rapamycin, a specific inhibitor of mTOR. The reported data show that mTOR activation requires the activation of the signaling cascade PI3K/PDK1/Akt, as assessed by the effect of the PI3K inhibitors wortmannin and Ly294002 that block PDK1, Akt and mTOR phos- phorylation, and SMC proliferation, in agreement with previous reports $[5,8,9]$.

It may be noted that, in addition to the role of the PI3K/PDK1/Akt/mTOR pathway (reported here), oxLDL-induced SMC proliferation requires also another signaling cascade involving metalloproteases (MT1-MMP and MMP2) and sphingolipid mediators generated by the neutral sphingomyelinase- 2 and sphingosine kinase-1 [28]. All these data suggest that the two signaling pathways (PI3K/mTOR and sphingolipid pathways) converge towards and activate cell cycle progression. Moreover, PI3K activation inhibits the pro-apoptotic effect of moderate concentration of oxLDL [5], and plays thereby a major role to allow expressing oxLDLinduced SMC proliferation.

The polyphenolic phytoalexin resveratrol, has been shown to reduce SMC and cardiac fibroblast proliferation [18,23] and to inhibit PI3K [29]. As the reported data shown that PI3K/Akt/mTOR pathway is required for oxLDL-induced SMC proliferation, this led us to investigate whether resveratrol inhibited the activation of the PI3K/Akt/mTOR signaling and SMC proliferation by oxLDL. As expected, resveratrol $(25 \mu \mathrm{M})$ blocked effectively the activation of the PI3K/Akt/mTOR signaling pathway and SMC proliferation. Of note, the resveratrol concentration used here was in the middle of those utilized in the literature. For instance, inhibitory concentration of resveratrol ranged from 5 to $100 \mu \mathrm{M}$ on cultured fibroblasts, cardiomyocytes, rhabdomyosarcoma cells, HepG2 [23,29-31]. It is to note that this concentration of resveratrol $(25 \mu \mathrm{M})$ did not induce any apoptotic effect, while higher concentration $(50 \mu \mathrm{M})$ was slightly toxic for SMC. These data are in agreement with the fact that resveratrol, like many anti-oxidants, is either a pro-survival or a pro-apoptotic agent, depending on the dose, the cell type, and the nature of the stimulus [25].

In our experimental system, resveratrol, as well as the other tested anti-oxidants NAC and Trolox, inhibited the early rise of intracellular ROS elicited by oxLDL, but it is likely that the inhibition of PDK1 phosphorylation, was not due to its anti-oxidant properties, since NAC and Trolox had no effect on PDK1. These results are in agreement with the lack of effect of anti-oxidants on the first early steps of oxLDL mitogenic signaling, such as EGFR phosphorylation and PI3K activation [21].

The reported data show that the oxLDL-induced activation of $\mathrm{PI} 3 \mathrm{~K} / \mathrm{Akt} / \mathrm{mTOR}$ is strongly inhibited by resveratrol. This is consistent with several reports that point out the crucial role of 
the inhibition of PI3K/Akt in the biological effect of resveratrol $[29,32,33]$. This inhibitory effect has been associated with the signaling through estrogen receptor ERalpha [32]. Another mechanism proposed by [30] suggests that resveratrol activates Shp2, thereby preventing interaction between Gab1 and PI3K that is necessary for further signal transduction.

Other mechanisms evoked by resveratrol could involve a direct inhibition of PDK1, or an increased PTEN activity resulting in active Akt dephosphorylation [34]. It is to note that mTOR phosphorylation by oxLDL requires neither ERK1/2 nor the other mitogenic signaling evoked by oxLDL, such as the sphingolipid pathway, since inhibiting these pathways did not affect the activation of PI3K and Akt (data not shown), in agreement with Auge et al. [5].

Resveratrol appears as a potent modulator of the balance proliferation/apoptosis characteristic of oxLDL and oxidative stress, since it is able to reverse oxLDL-induced apoptosis of endothelial cells [13], and to increase the oxidative stress resistance of vascular cells [14], but it may also inhibit oxLDL-induced SMC proliferation, in agreement with previously reported data [18]. It is to note that resveratrol also inhibited ERK1/2 phosphorylation in our system, which takes place in the general inhibitory effect of this molecule on DNA synthesis elicited by oxLDL. These data are in agreement with reports showing that resveratrol inhibits MAPK activation elicited by various agents including growth factors (PDGF) [16], angiotensin II [17,35] and oxLDL [18]. At the opposite, some reports indicate that ERK1/2 phosphorylation induced by EGF is not affected by resveratrol [16]. These discrepancies could also result from differences in the cell type, the nature of the stimulus and the concentration of the polyphenol. For instance, in human prostate carcinoma DU145 cells, low doses of polyphenol-rich grape seed extracts inhibited EGF-induced ERK1/2 phosphorylation, whereas higher doses induced an increase [15]. Here we show that the PI3K/Akt/mTOR pathway is not involved in ERK1/2 phosphorylation, as previously reported in [5]. These data indicate that other mitogenic pathways are activated by oxLDL, and involved in ERK1/2 phosphorylation, independently of the PI3K/Akt system, and in agreement with our previous data showing an involvement of the metalloproteinase/sphingolipid pathway in oxLDL- and TNF $\alpha$-mediated SMC proliferation [28,19]. As a matter of fact, preliminary experiments indicate that the sphingolipid pathway is also inhibited by resveratrol, thereby inhibiting in turn ERK1/2 phosphorylation, independently of the PDK1/Akt/mTOR system.

Since AMPK is known to be activated by resveratrol [26] and activated AMPK is able to inhibit mTOR (mediated by two mechanisms, TSC2 phosphorylation and raptor phosphorylation) [27], and may participate to the anti-atherogenic effect of resveratrol [26,31], we investigated whether AMPK was required for the anti-mitogenic effect of resveratrol in our experimental model system. Under the used conditions, AMPK was activated by resveratrol, but not by oxLDL. This was unexpected, because AMPK is able to respond to oxidative stress [36] and oxLDL trigger a cellular oxidative stress. Moreover, unexpectedly, oxLDL inhibited AMPK activation by resveratrol. This led us to conclude that, in spite AMPK is activated by resveratrol under basal conditions (i.e. in the absence of oxLDL), it is not activated in the presence of oxLDL, thus indicating that AMPK is not involved in the inhibitory effect of resveratrol on PI3K/Akt/mTOR activation by oxLDL.

From a general point of view, resveratrol exhibits various potentially anti-atherogenic properties, including anti-oxidant effect and an ability to modulate major signaling pathways, such as the PI3K/PDK1/Akt/mTOR system. Therefore, resveratrol is susceptible of slowing down the formation of foam cells and fatty streaks in early atherosclerotic lesions (via its anti-oxidant effect), which indi- rectly limit the migration and proliferation of SMC in the intima. On the other hand, the inhibitory effect of resveratrol on the PI3K/Akt/mTOR activation and SMC proliferation, suggests that this polyphenol could help to limit the deregulated SMC proliferation occurring in extensive atherosclerotic arterial stenosis and in postangioplasty restenosis.

\section{Acknowledgments}

This work was supported by Inserm, Paul Sabatier University, Agence Nationale pour la Recherche (ANR-05-PCOD-019-01-LiSA), FCT (Proj. POCTI/35019/AGR/2000 and POCI/AGR/599919/2004). Paula Brito is a recipient of the grant SFRH/BD/7986/2001.

\section{References}

[1] Lusis AJ. Atherosclerosis. Nature 2000;407:233-41.

[2] Berliner JA, Heinecke JW. The role of oxidized lipoproteins in atherogenesis. Free Radic Biol Med 1996;20:707-27.

[3] Auge N, Escargueil-Blanc I, Lajoie-Mazenc I, et al. Potential role for ceramide in mitogen-activated protein kinase activation and proliferation of vascular smooth muscle cells induced by oxidized low density lipoprotein. J Biol Chem 1998;273:12893-900.

[4] Auge N, Negre-Salvayre A, Salvayre R, Levade T. Sphingomyelin metabolites in vascular cell signaling and atherogenesis. Prog Lipid Res 2000;39: 207-29.

[5] Auge N, Garcia V, Maupas-Schwalm F, Levade T, Salvayre R, Negre-Salvayre A. Oxidized LDL-induced smooth muscle cell proliferation involves the EGF receptor/PI-3 kinase/Akt and the sphingolipid signaling pathways. Arterioscler Thromb Vasc Biol 2002;22:1990-5.

[6] Nave BT, Ouwens M, Withers DJ, Alessi DR, Shepherd PR. Mammalian target of rapamycin is a direct target for protein kinase B: identification of a convergence point for opposing effects of insulin and amino-acid deficiency on protein translation. Biochem J 1999;344(Pt 2):427-31.

[7] Shaw RJ, Cantley LC. Ras, PI(3)K and mTOR signalling controls tumour cell growth. Nature 2006;441:424-30.

[8] Sekulic A, Hudson CC, Homme JL, et al. A direct linkage between the phosphoinositide 3-kinase-AKT signaling pathway and the mammalian target of rapamycin in mitogen-stimulated and transformed cells. Cancer Res 2000;60:3504-13.

[9] Wullschleger S, Loewith R, Hall MN. TOR signaling in growth and metabolism. Cell 2006;124:471-84.

[10] Pace-Asciak CR, Hahn S, Diamandis EP, Soleas G, Goldberg DM. The red wine phenolics trans-resveratrol and quercetin block human platelet aggregation and eicosanoid synthesis: implications for protection against coronary heart disease. Clin Chim Acta 1995;235:207-19.

[11] Brito P, Almeida LM, Dinis TC. The interaction of resveratrol with ferrylmyoglobin and peroxynitrite; protection against LDL oxidation. Free Radic Res 2002;36:621-31.

[12] Brito PM, Mariano A, Almeida LM, Dinis TC. Resveratrol affords protection against peroxynitrite-mediated endothelial cell death: a role for intracellular glutathione. Chem Biol Interact 2006;164:157-66.

[13] Ou HC, Chou FP, Sheen HM, Lin TM, Yang CH, Huey-Herng Sheu W. Resveratrol a polyphenolic compound in red wine, protects against oxidized LDL-induced cytotoxicity in endothelial cells. Clin Chim Acta 2006;364:196-204.

[14] Ungvari Z, Orosz Z, Rivera A, et al. Resveratrol increases vascular oxidative stress resistance. Am J Physiol Heart Circ Physiol 2007;292:H2417-2424.

[15] Tyagi A, Agarwal R, Agarwal C. Grape seed extract inhibits EGF-induced and constitutively active mitogenic signaling but activates JNK in human prostate carcinoma DU145 cells: possible role in antiproliferation and apoptosis. Oncogene $2003 ; 22: 1302-16$.

[16] Godichaud S, Si-Tayeb K, Auge N, et al. The grape-derived polyphenol resveratrol differentially affects epidermal and platelet-derived growth factor signaling in human liver myofibroblasts. Int J Biochem Cell Biol 2006;38:629-37.

[17] Haider UG, Sorescu D, Griendling KK, Vollmar AM, Dirsch VM. Resveratrol suppresses angiotensin II-induced Akt/protein kinase B and p70 S6 kinase phosphorylation and subsequent hypertrophy in rat aortic smooth muscle cells. Mol Pharmacol 2002;62:772-7.

[18] Liu Y, Liu G. Isorhapontigenin and resveratrol suppress oxLDL-induced proliferation and activation of ERK1/2 mitogen-activated protein kinases of bovine aortic smooth muscle cells. Biochem Pharmacol 2004;67:777-85.

[19] Tellier E, Negre-Salvayre A, Bocquet B, et al. Role for furin in tumor necrosis factor alpha-induced activation of the matrix metalloproteinase/sphingolipid mitogenic pathway. Mol Cell Biol 2007;27:2997-3007.

[20] Auge N, Pieraggi MT, Thiers JC, Negre-Salvayre A, Salvayre R. Proliferative and cytotoxic effects of mildly oxidized low-density lipoproteins on vascular smooth-muscle cells. Biochem J 1995;309(Pt 3):1015-20.

[21] Vacaresse N, Vieira O, Robbesyn F, Jurgens G, Salvayre R, Negre-Salvayre A. Phenolic antioxidants trolox and caffeic acid modulate the oxidized LDL-induced EGF-receptor activation. Br J Pharmacol 2001;132:1777-88. 
[22] Burgering BM, Freed E, van der Voorn L, McCormick F, Bos JL. Platelet-derived growth factor-induced p21ras-mediated signaling is independent of plateletderived growth factor receptor interaction with GTPase-activating protein or phosphatidylinositol-3-kinase. Cell Growth Differ 1994;5:341-7.

[23] Olson ER, Naugle JE, Zhang X, Bomser JA, Meszaro JG. Inhibition of cardiac fibroblast proliferation and myofibroblast differentiation by resveratrol. Am J Physiol Heart Circ Physiol 2005;288:H1131-1138.

[24] Oak MH, El Bedoui J, Schini-Kerth VB. Antiangiogenic properties of natural polyphenols from red wine and green tea. J Nutr Biochem 2005;16:1-8.

[25] Holme AL, Pervaiz S. Resveratrol in cell fate decisions. J Bioenerg Biomembr 2007;39:59-63.

[26] Zang M, Xu S, Maitland-Toolan KA, et al. Polyphenols stimulate AMP-activated protein kinase, lower lipids, and inhibit accelerated atherosclerosis in diabetic LDL receptor-deficient mice. Diabetes 2006;55:2180-91.

[27] Gwinn DM, Shackelford DB, Egan DF, et al. AMPK phosphorylation of raptor mediates a metabolic checkpoint. Mol Cell 2008;30:214-26.

[28] Auge N, Maupas-Schwalm F, Elbaz M, et al. Role for matrix metalloproteinase-2 in oxidized low-density lipoprotein-induced activation of the sphingomyelin/ceramide pathway and smooth muscle cell proliferation. Circulation 2004; 110:571-8.

[29] Frojdo S, Cozzone D, Vidal H, Pirola L. Resveratrol is a class IA phosphoinositide 3-kinase inhibitor. Biochem J 2007;406:511-8.
[30] Haider UG, Roos TU, Kontaridis MI, et al. Resveratrol inhibits angiotensin II- and epidermal growth factor-mediated Akt activation: role of Gab1 and Shp2. Mol Pharmacol 2005;68:41-8.

[31] Chan AY, Dolinsky VW, Soltys CL, et al. Resveratrol inhibits cardiac hypertrophy via AMP-activated protein kinase and Akt. J Biol Chem 2008;283:24194201.

[32] Pozo-Guisado E, Lorenzo-Benayas MJ, Fernandez-Salguero PM. Resveratrol modulates the phosphoinositide 3-kinase pathway through an estrogen receptor alpha-dependent mechanism: relevance in cell proliferation. Int J Cancer 2004;109:167-73.

[33] Cullen JP, Morrow D, Ji Y, et al. Resveratrol inhibits expression and binding activity of the monocyte chemotactic protein-1 receptor, CCR2, on THP-1 monocytes. Atherosclerosis 2007; 195:e125-133.

[34] Hill MM, Hemmings BA. Inhibition of protein kinase B/Akt implications for cancer therapy. Pharmacol Ther 2002;93:243-51.

[35] Chao HH, Juan SH, Liu JC, et al. Resveratrol inhibits angiotensin II-induced endothelin-1 gene expression and subsequent proliferation in rat aortic smooth muscle cells. Eur J Pharmacol 2005;515:1-9.

[36] Jung SN, Yang WK, Kim J, et al. Reactive oxygen species stabilize hypoxiainducible factor- 1 alpha protein and stimulate transcriptional activity via AMP-activated protein kinase in DU145 human prostate cancer cells. Carcinogenesis 2008;29:713-21. 